\title{
A Graphical Method of Determining Ocean Currents.
}

\section{Mituyo OKADA}

(The Imperial Fisheries Institute, Tôkyô)

'The writer ${ }^{1)}$ has previously studied on the application of KNUDsEn's law to determine the steady surface current in coastal waters based on the observation of salinity, $S$, and soluble silicate content, $P$. In that case, though the direction without distinguishing sense and the relative spced of the current could be determined, no means were given for the determination of the sense and the absolute magnitude of the current. In this paper, it is studied whether or not the latter two can be determined under the assumptions:-

(1) That the current, as well as the distribution of salinity and silicate, is in a stationary state; (2) that the vertical component of flow multiplied by the vertical gradient of $S$ or $P$ is negligible as compared with the horizontal components multiplied by the horizontal gradient of $S$ or $P$; (3) that the coefficient of diffusion due to turbulence is the same for $S$ and $P$, and also for the temperature ${ }^{2)}, \theta$; (4) that the variation of density with temperature and salinity of the sea water is, of course, negligible; and (5) that the dilution or concentration of sea water due to precipitation or evaporation at the surface, or otherwise, due to biochemical processes is also negligible.

Consider a thin surface layer, in which the $x$ - and $y$-axes of rectangular coordinates are taken horizontally and the depth is denoted by $z$. Denote the $x$ - and $y$-components of the steady current at $(x, y, z)$ by $u^{\prime}$ and $v^{\prime}$ respectively. Then, from the assumptions given above, we get

and

$$
\begin{aligned}
& u^{\prime} \frac{\partial S}{\partial x}+v^{\prime} \frac{\partial S}{\partial y}=k^{\prime} \Delta S+k \frac{\partial^{2} S}{\partial z^{2}} . \\
& u^{\prime} \frac{\partial P}{\partial x}+v^{\prime} \frac{\partial P}{\partial y}=k^{\prime} \Delta P+k \frac{\partial^{2} P}{\partial z^{2}},
\end{aligned}
$$

where $\Delta \equiv \frac{\partial^{2}}{\partial x^{2}}+\frac{\partial^{2}}{\partial y^{2}}$, and the coefficients of diffusivity, $k^{\prime}$ and $k$, are different from $^{3)}$ or equal to each other. In practice, it is generally the case that $\Delta S \ll \frac{\partial^{2} S}{\partial z^{2}}$, and $\Delta P \ll \frac{\partial^{2} P}{\partial z^{2}}$, and it can safely be assumed that the first term on

1) OKada, M.: Estimation of steady surface current in coastal waters based on the observation of salinity and soluble kilicate content. Bull. Jap. Soc. Sci. Fish., 3(3), 1934, $121 \sim 124$.

2) Strictly speaking, for the potential temperature.

3) For example, see Witing, R.: Zur Bestimmung der Mischung im Meere. Soc. Sci. Fennica. Comment. Phys.-Mathem., 7 (2), 1933. 1 30. 
the right-band side of both equations is negligible as compared with the second. Reducing the thickness of the surface layer and taking the last assumption (5) into consideration, we get, instend of the above equations, the following two :-

and

$$
\begin{aligned}
& u S_{x}+v S_{y}=\frac{k}{h} S_{z}, \\
& u P_{x}+v P_{y}=\frac{k}{h} P_{z},
\end{aligned}
$$

where $h$ is the thickness of the layer, $S_{x}=\frac{\partial \bar{S}}{\partial x}, S_{y}=\frac{\partial \bar{S}}{\partial y}, S_{z}=\left(\frac{\partial S}{\partial z}\right)_{z=h}, P_{x}=\frac{\partial \bar{P}}{\partial x}$, $P_{y}=\frac{\partial \bar{P}}{\partial y}$, and $P_{z}=\left(\frac{\partial P}{\partial z}\right)_{z-h}, u, v, \bar{S}$ and $\bar{P}$ being mean values of $u^{\prime}, v^{\prime}, S$ and $P$ respectively at the depths $z=0$ to $z=h$.

If we represent the horizontal distribution of $\bar{S}$ and $\bar{P}$ in the layer by drawing the iso-lines of them, and take the $x$-axis in the direction of the tangent line at an assigned point, $P$, to the iso-line of $S$ passing through $P$, then at $\mathrm{P}, S_{x}=0$. Consequently,

$$
v=\frac{k}{h} \cdot \frac{S_{z}}{S_{y}}, \quad \text { and } \quad u=\frac{k}{h} \cdot \frac{S_{y} P_{z}-S_{z} P_{y}}{S_{y} P_{x}}
$$

Similarly, when the $x$-axis is taken in the direction of the iso-line of $P$,

$$
v=\frac{k}{h} \cdot \frac{P_{z}}{P_{y}}, \quad \text { and } \quad u=\frac{k}{h} \cdot \frac{P_{y} S_{z}-P_{z} S_{y}^{\prime}}{P_{y} S_{x}}
$$

Thus, if the value of $k$ is given in any other way, we can determine the direction and the absolute magnitude of the current by means of the equations (1) or (2).

Draw, in a chart, the iso-lines of both $\bar{S}$ and $\bar{P}$, grading them uniformly with a suitable units. On the other hand, evaluate $S_{z}$ and $P_{z}$ from the observational data and express them in terms of those units of $S$ and $P$, just mentioned, and some common unit of $z$. It is preferable to draw the isolines of $S_{z}$ and $P_{z}$ in another chart. At the point of intersection of a $S$-line and a $P$-line on the first chart, draw the two arrows, $\mathcal{S}$ and $\mathfrak{P}$ (Fig. 1), which start from the point at right angles to the respective iso-lines and end on the next iso-lines, having greater values of $S$ or $P$. Then, reading off the values of $S_{z}$ and $P_{z}$, if necessary from the second chart, construct the two vectors $S_{z} S$ and

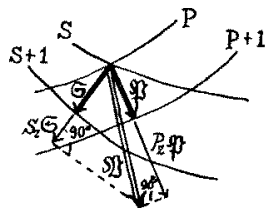

Fig. 1. $P_{z}$. Since $|\mathfrak{S}|$ and $|\mathfrak{P}|$ are nearly proportional to $\frac{1}{S_{y}}$ and $\frac{1}{P_{y}}$ in the first equations of (1) and (2), where the positive direction of $y$ is taken in the same direction as $\mathfrak{S}$ or $\mathfrak{P}$, the last two vectors are proportional to $v$ in (1) and 
that in (2) respectively. The vector, $\mathfrak{B}$, representing the resultant horizontal current can easily be constructed graphically from the two vectors, just obtained, as in Fig. 1.

Fig. 2 shows an example, of which the data, same as what were dealt with in the previous paper, were got from the observation, made on Sept. 6 to 8,1933 , in Sagami Bay ${ }^{1}$. $h$ was chosen to be $25 \mathrm{~m}$, and the isolines of $S$ and $P$ were drawn with the mean values of them at $0 \mathrm{~m}$ and at $25 \mathrm{~m}$, while the vertical gradients at $25 \mathrm{~m}$, were calculated from the differences of values at $0 \mathrm{~m}$ from those at $50 \mathrm{~m}$ (Tab. 1). The double arrows in Fig. 2 indicate the horizontal flow estimated by the present method, whilesingle arrows by the previous method. A satisfactory coincidence is seen between the results obtained by the two methods in respect of the di-

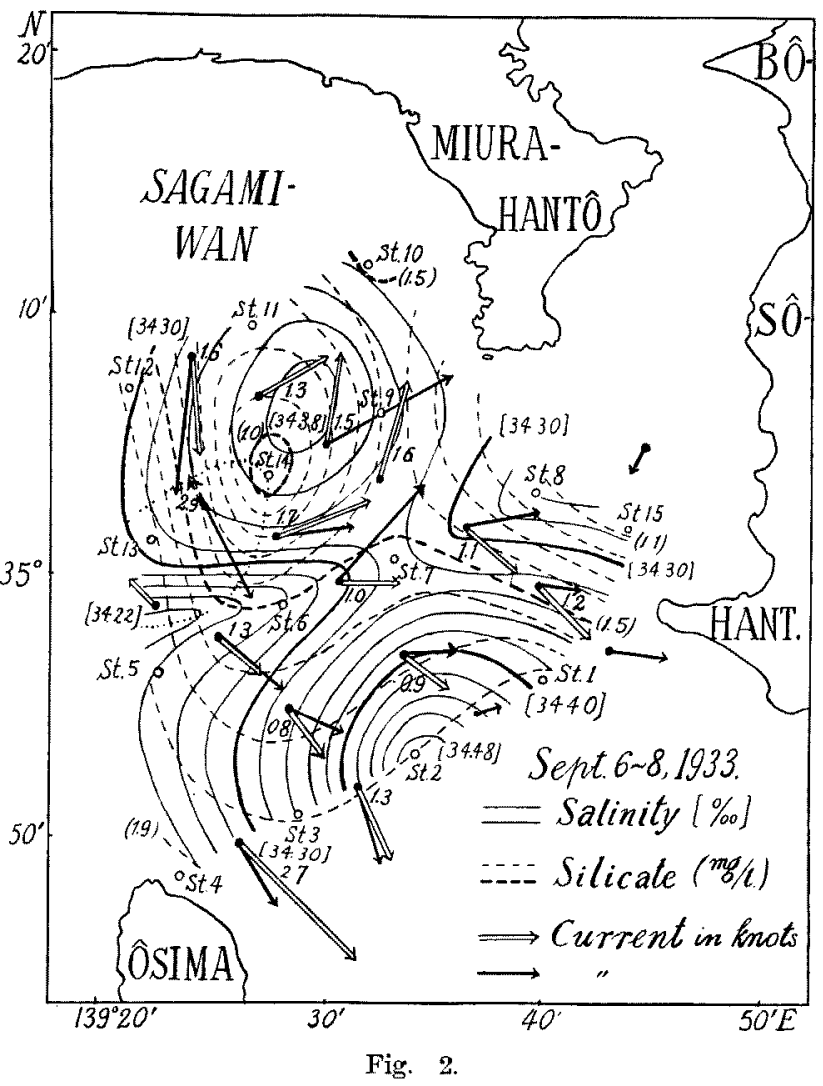
rection and the relative speed of the flow, except in some region ${ }^{2)}$, as, for example, that indicated by a dotted line in Fig. 2. To estimate the order of maguitude of the ipeed, the value of $k$ was determined from the averaged temperatures $\vec{\theta}$ observed at the same time and those observed after about 20

Table 1. The vertical gradients of salinity and silicate content at $25 \mathrm{~m}$.

\begin{tabular}{|c|ccccccccccccccc|}
\hline Stations & 1 & 2 & 3 & 4 & 5 & 6 & 7 & 8 & 9 & 10 & 11 & 13 & 13 & 14 & 15 \\
\hline$S_{z}\left(10^{-30} / \mathrm{m} / \mathrm{m}\right)$ & 7.6 & 6.4 & 8.4 & 8.6 & 9.2 & 11.0 & 8.8 & 8.4 & 7.0 & 7.6 & 7.6 & 9.0 & 7.0 & 8.8 & 6.2 \\
$P_{z}\left(10^{-2} \mathrm{mg} / \mathrm{l} / \mathrm{m}\right)$ & 1.7 & 2.8 & 1.5 & 1.7 & 0.7 & 02 & 0.6 & 1.7 & 1.4 & 1.5 & 1.5 & 1.8 & 1.4 & 1.8 & 1.2 \\
\hline
\end{tabular}

1) The data are given in Semiann. Rep. Oceanogr. Invest,, (53), 1934, p. 52 and p. 278.

2) No acceptable explanation for the existence of such regions of discrepancy can be given from the present limited knowledge of observation. 
Table 2. The vertical distribution of the average temperatures in the region.

\begin{tabular}{|c|c|c|c|c|}
\hline \multicolumn{3}{|c|}{ Dates } & 6 to 7 , Sept., $\left.1933^{1}\right)$ & 27, Sept., $1933^{2}$ \\
\hline \multicolumn{3}{|c|}{ Stations } & St. $1,2,3,4,7,8$ and 9 (Fig. 1 ) & $\begin{array}{l}\text { St. } 23,2,3,4,-, 21 \text { and } 17 \text { of the } \\
\text { Imper. Fish. Exper. Station. }\end{array}$ \\
\hline $\begin{array}{c}\text { Aver. } \\
" \prime \\
"\end{array}$ & $\begin{array}{c}\text { temp. a } \\
" \prime \\
"\end{array}$ & $\begin{array}{ll}\text { at } & 0 \mathrm{~m} \\
\text { " } & 25 \\
\text { " } & 50\end{array}$ & $\begin{array}{l}25.24^{\circ} \mathrm{C} \\
19.48 \\
16.66\end{array}$ & $\begin{array}{l}25.04^{\circ} \mathrm{C} \\
24.83 \\
22.46\end{array}$ \\
\hline
\end{tabular}

days in the sime region by the hydrographers of the Imperial Fisheries Experimental Station, Tôkyô (Tab. 2). The equation ${ }^{3)}$,

$$
\frac{\partial \bar{\theta}}{\partial t}=k \frac{\partial^{2} \bar{\theta}}{\partial z^{2}}
$$

was made use of, and the value $k=50 \mathrm{~cm}^{2} / \mathrm{sec}$ was obtained for 0 to $50 \mathrm{~m}$. This is of the same order, though about five times as large, as what was obtained previously by $\mathrm{M}$. $\mathrm{UDA}^{4)}$ in the same way for the month of September, in the wider region of Sagami Bay. With that value of $k$, the speed was estimated to be mostly in the range 0.8 to $2.7 \mathrm{knots}$ (Fig. 2). Mr. UDA has kindly informed the writer that the speed of the surface current in the middle part of the Bay, measured with current drags in the winter of 1933-'34 was of the order of 2 knots or a little more.

Thus, we can conclude that the method can be employed, at least as the first approximation, to estimate the horizontal flow of the surface layer. The method fails where the iso-lines of two kinds ran parallel or nearly parallel to each other, or where small whirls escaped from the meshes of observational stations.

The writer's cordial thanks are due to Dr. M. TAUTI and Mr. M. UDA, for the valuable suggestions and informations given to lim.

1) Semiann. Rep. Ocean. Invest., (53), 1934, p. 52.

2) Dit., p. 53 .

3) From the values of the speed obtained below and from the distribution of $\theta$ observed, we know that $u \frac{\partial \theta}{\partial x}$ is of the same order as $\frac{\partial \theta}{\partial t}$, but when the average is taken in wide range, the terms, $u \frac{\partial \theta}{\partial x}$ and $v \frac{\partial \theta}{\partial y}$, become negligible.

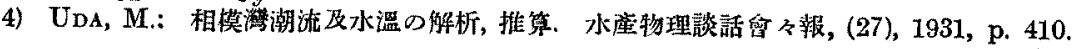

